\title{
Cuadros depresivos en la fase aguda del ictus isquémico. ¿Una reacción de duelo?
}

\author{
Post-stroke depression. A grief reaction?
}

RESUMEN: Realizamos un estudio prospectivo para valorar la depresión en la fase aguda del ictus, y hallamos similitudes con una reacción de duelo.

PALABRAS CLAVE: depresión postictus, duelo.
ABSTRACT: We performed a prospective research with the aim to assess the depression in acute stroke, and we identified resemblances between poststroke depression and the grief.

KEY WORDS: poststroke depression, grief.

\section{Introducción.}

La American Heart Associaton incluye las alteraciones afectivas dentro de los seis campos de discapacidad generada por accidentes cerebrovasculares, entre las cuales, la complicación psiquiátrica más frecuente son los cuadros depresivos, estimándose en un 50\% de los pacientes en el periodo agudo postictus y en el $30 \%$ de pacientes ictados tratados de forma ambulatoria $(1 ; 2)$. Desde que se describiera por primera vez la relación entre depresión y patología vascular a principios del siglo XX (3) hasta la actualidad, se han llevado a cabo numerosos estudios en un intento de dilucidar la etiopatogenia de la depresión postictus. Entre los hallazgos más consistentes de los diversos estudios sobre el tema, se encuentran:

- La probabilidad de sufrir una depresión postictus es mayor entre los tres primeros meses y los doce meses tras el ictus y comienza a reducirse a partir de entonces $(4 ; 5)$.

- En la mayoría de los estudios, no se encuentra correlación significativa entre la presencia de cuadro depresivo y la etiología y extensión del ictus (5) y, entre los que sí la han encontrado, los resultados no son concluyentes $(8 ; 9)$

- Clínicamente, no es posible distinguir la depresión tras un ictus de una depresión funcional (10).

Partiendo de estos datos, considerando que la pauta temporal anteriormente expuesta sobre la depresión postictus coincide con la duración de la elaboración de un duelo no patológico, y apoyándonos en las teorías psicológicas al respecto 
de la depresión tras el ictus, nos planteamos la posibilidad de que este estado afectivo se trate de una reacción adaptativa de duelo.

En los últimos años, el concepto de duelo se ha ampliado y ya no se entiende únicamente como el dolor ante la muerte de un ser querido, sino que implica la reacción psicológica ante una pérdida significativa para nuestra vida, entendiendo por pérdida: la privación de algo que se ha tenido, fracaso para conservar algo de valor personal o una reducción considerable de algún objeto o proceso (12). A lo largo de la vida, todo ser humano se encuentra en numerosas situaciones en las que tiene que afrontar la elaboración de un duelo: fallecimiento de un ser querido, separación sentimental de una pareja, de un amigo, divorcio, pérdida de un empleo, emigración, mudanza de domicilio y, por supuesto, también la enfermedad como pérdida de salud y/o capacidad funcional ya sea propia o en seres queridos $(12 ; 13 ; 14)$. La Clasificación Internacional de Trastornos Mentales y del Comportamiento CIE-10 incluye las reacciones de duelo en los Trastornos de Adaptación (F43.2), afirmando que en éstos "Se trata de estados de malestar subjetivo acompañados de alteraciones emocionales que, por lo general, interfieren con la actividad social y que aparecen en el periodo de adaptación a un cambio biográfico significativo o a un acontecimiento vital estresante" (15) y que el malestar subjetivo debe aparecer durante el primer mes tras la situación estresante identificable. Ya en 1948, Goldstein explicó la depresión tras un ictus como una reacción catastrófica ante la incapacidad del sujeto para hacer frente a sus déficits (16) y con posterioridad, también otros autores abogan por considerar que la depresión postictus no es sino una reacción del paciente al percibirse incapaz de afrontar sus limitaciones $(17 ; 18 ; 19)$.

Siguiendo esta línea, entendemos que sufrir un ictus isquémico es un acontecimiento vital estresante a cuyas consecuencias el paciente ha de adaptarse y consideramos que, entre dichas consecuencias, se encuentran diversos tipos de pérdida (de salud, de capacidad, de funcionalidad, separación de la familia por estar hospitalizado, etc). Desde esta concepción, nos planteamos un estudio descriptivo con dos objetivos fundamentales: explorar la clínica neurológica y depresiva, en caso de que la hubiera, en pacientes con ictus isquémico e identificar posibles variables asociadas a fin de comprobar si se da un perfil característico que nos permita someter a discusión si la depresión postictus pudiera tratarse de una reacción de duelo.

\section{Material y Método.}

Selección de pacientes:

Realizamos un estudio prospectivo sobre una muestra constituida por cin- 
ORIGINALES Y REVISIONES

cuenta pacientes que, tras sufrir un ictus isquémico, fueron ingresados de forma consecutiva en la Sección de Neurología del Hospital Universitario de Gran Canaria Dr. Negrín a través del Servicio de Urgencias. En este servicio, se ingresan todos los ictus de menos de una semana de evolución excepto en los casos en los que previamente exista un nivel de dependencia funcional que condicione el beneficio que se pueda obtener de la hospitalización. El Hospital Universitario de Gran Canaria Doctor Negrín es el centro de referencia para la zona norte de la Isla de Gran Canaria dado que éste abarca una población de 450.000 habitantes.

Definición y clasificación de ictus isquémico:

Se incluyen en la definición de ictus isquémico todos los episodios de clínica neurológica focal con una duración mayor de 24 horas, o bien con una duración menor de 24 horas pero en los que se observan una lesión compatible con isquemia en las pruebas de neuroimagen. Se excluyeron de esta definición todos aquellos pacientes en los que (a) las pruebas de neuroimagen hubieran puesto de manifiesto la presencia de otra causa que justificara la clínica neurológica focal (hemorragias, abscesos, tumores...) y (b) las pruebas neurofisiológicas fueran compatibles con un origen comicial del evento.

La clasificación clínica se realizó según la escala de la Oxforshire Community Stroke Project (OCSP), que distingue entre TACI (infarto total de la circulación anterior), PACI (infarto parcial de la circulación anterior), LACI (infarto lacunar) y POCI (infarto de circulación posterior). La etiología del ictus se estudió según la clasificación TOAST modificada. Las cinco categorías de la clasificación TOAST son: 1) ictus de origen aterotrombótico (presencia de una estenosis carotídea $50 \%$ ipsilateral al ictus o una estenosis carotídea $<50 \%$ ipsilateral al ictus si se acompaña de dos o más factores de riesgo vacular); 2) ictus de origen cardioembólico (presencia de una cardiopatía potencialmente embolígena y ausencia de otras causas que lo justifiquen); 3 ) ictus lacunar (presentación con alguno de los síndromes lacunares clásicos y lesión $<15 \mathrm{~mm}$ en las pruebas de neuroimagen o pruebas de neuroimagen normales, sin otra causa que lo justifique);4 ictus de causa inhabitual y 5) ictus indeterminado (no se encuentra causa a pesar de estudio exhaustivo o bien se encuentra más de una causa que justifique el ictus). En todos los casos se había realizado TAC craneal en el momento del ingreso. En nuestro centro se realiza ecografía doppler de troncos supraaórticos en todos los pacientes con sospecha de AIT o II. Se realiza ecocardiograma en aquellos casos en los que exista un antecedente de cardiopatía o algún indicio en la anamnesis o exploración física que sugiera una cardiopatía potencialmente embolígena. El estudio con doppler transcraneal se ha ido instaurando progresivamente, de manera que actualmente sólo los pacientes que ingresan en nuestra unidad de ictus lo tienen en el protocolo de estudio.

La gravedad del ictus isquémico se midió mediante la escala NIHSS en el 
momento del ingreso en Neurología (12-36 horas después de sufrir el ictus).

Evaluación del estado de ánimo:

Para establecer la presencia de cuadro depresivo, seguimos los criterios diagnósticos establecidos por las clasificaciones internacionales formales de los trastornos mentales y del comportamiento DSM-IV-TR y CIE-10, aunque prescindimos de los criterios temporales así como de la distinción que en las mismas se hace de episodio depresivo mayor y trastorno del estado de ánimo debido a enfermedad médica. Así pues, consideramos como cuadro depresivo la constelación de signos y síntomas depresivos desde una perspectiva transversal. La evaluación de la ausencia/ presencia de cuadros depresivos así como de la gravedad de los mismos se llevó a cabo mediante la Escala de Depresión de MontgomeryAsberg (MADRS), y los puntos de corte establecidos por la misma: 0-6 puntos, sin depresión; 7-19 puntos, depresión menor; 20-34 puntos, depresión moderada; y 35-60 puntos, depresión grave. Hemos elegido este instrumento de evaluación porque consta de sólo diez ítems sencillos de responder, con lo que se reduce la probabilidad de fatigar al paciente en el proceso de administración y, por otro lado, presenta la ventaja de no estar contaminada por ítems que evalúan ansiedad; además, aunque el MADRS tiene varios ítems de carácter somático, éstos son referidos a tensión interior y cambios en los hábitos de alimentación y sueño, y no incluyen afectación psicomotora.

Por otra parte, elaboramos una ficha de recogida de datos en la que recogimos no sólo variables clínicas (sexo, edad, fecha de ingreso, localización y extensión del ictus, nivel de NIH, cuáles eran los síntomas depresivos en caso de que los hubiera, gravedad del cuadro depresivo) sino que también evaluamos: 1) la variable "antecedentes personales de depresión" nominalmente con respuestas dicotómicas de Sí/No, a partir de la información aportada por el paciente y/o sus familiares; 2) la variable "síntomas depresivos previos al ictus", considerándose la existencia síntomas y signos de carácter depresivo los días, semanas y meses previos al inicio del ictus isquémico, también mediante respuestas dicotómicas Sí/No. La aplicación de estos instrumentos de evaluación del estado de ánimo se llevó a cabo durante los cinco primeros días tras sufrir el ictus.

Criterios de exclusión:

Se excluyeron del estudio aquellos pacientes que cumplían uno siguientes criterios: alteración de conciencia, afasia, deterioro cognitivo/demencia, abuso de sustancias, trastornos psiquiátricos que no fueran trastornos depresivos y aquéllos que hubieran sobrepasado el periodo agudo del ictus.

Análisis estadístico:

Para el análisis estadístico de los datos, se empleó el Programa SPSS 15.0 para Windows XP. Los cálculos del análisis univariado se operaron a través de media aritmética, el test de Chi Cuadrado con un error alfa de 0.5 y el estadístico 
ORIGINALES Y REVISIONES

U de Mann Whitney según el tipo de variables con el que se trabajara. Asimismo, se llevó a cabo cálculos de regresión logística para aquellas variables que resultaron significativas.

\section{Resultados.}

La muestra la componen 50 sujetos: 20 mujeres y 30 hombres, con una media de edad de 64,66 años. Hallamos que 26 pacientes (52\%) presentaban cuadro depresivo. De éstos (Gráfico I), ninguno presentaba depresión grave y de los 24 pacientes restantes que no mostraban depresión, 17 presentaban síntomas depresivos aislados aunque no llegaran a configurar la entidad de un cuadro depresivo completo. Esto nos deja con 43 pacientes que presentan sintomatología depresiva en la fase aguda del ictus isquémico frente a sólo 7 pacientes que no la presentaban.

\section{Gráfico 1}

Distribución por gravedad del cuadro depresivo

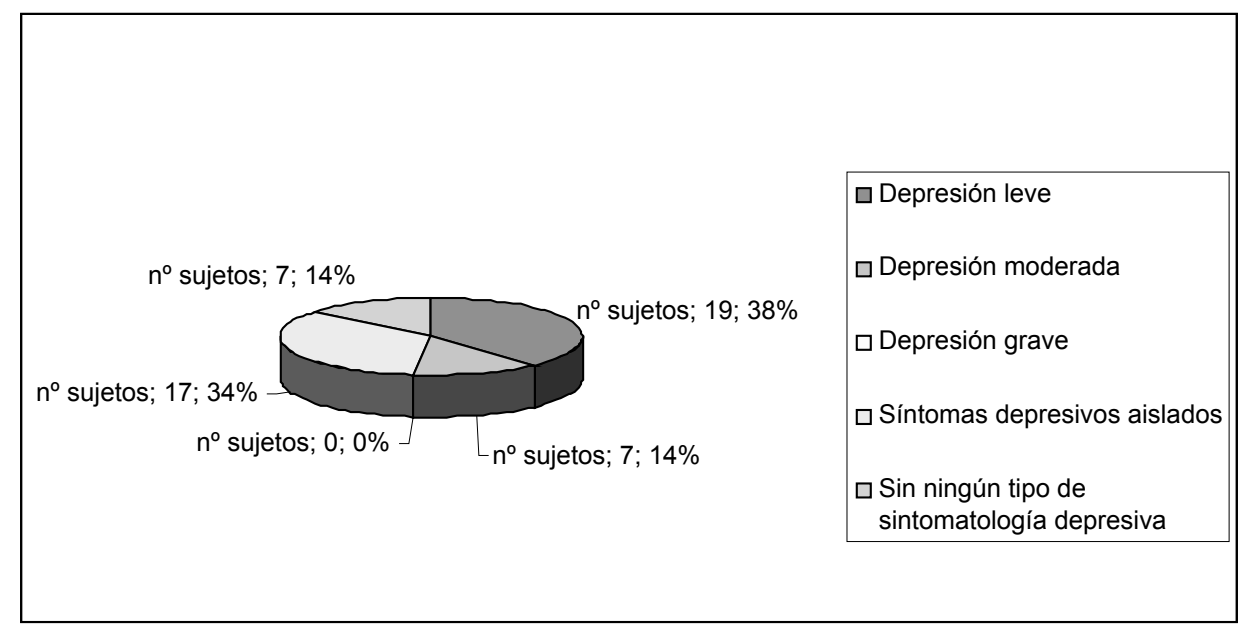

Como se observa en la Tabla 1, en nuestro estudio, no se encontró relación significativa entre la presencia de un cuadro depresivo postictus y la variable edad pero sí con la variable sexo femenino. Asimismo, la variable "síntomas depresivos previos al ictus" tampoco resultó significativa pero, en cambio, sí lo fue 
claramente la asociación entre presencia de cuadro depresivo tras el ictus y tener antecedentes personales de depresión.

En cuanto a la clínica neurológica, no se encontró relación significativa entre tener o no depresión postictus y la localización y extensión del ictus isquémico. Pero sí que resultó significativa la relación con la gravedad del ictus, medida con la escala NIHSS, hallándose en los pacientes ictados con cuadro depresivo un valor medio de 4,92 (3,04-6,8; IC 95\%) frente a un 2,21 (1,7-2,72; IC 95\%) en aquellos pacientes ictados que no padecieron un episodio depresivo después del ictus. Sin embargo, no se halló asociación entre nivel de NIHSS y gravedad del cuadro depresivo (leve/moderado).

Tabla 1

Variables asociadas a la presencia de depresión en la fase aguda del ictus isquémico. Estudio univariado.

\begin{tabular}{|l|c|c|c|}
\hline & $\begin{array}{c}\text { No depresión } \\
\mathrm{n}=24\end{array}$ & $\begin{array}{c}\text { Sí depresión } \\
\mathrm{n}=26\end{array}$ & OR \\
\hline Edad & $\begin{array}{c}63,50(57,9- \\
69,10 \mathrm{IC} 95 \%)\end{array}$ & $\begin{array}{c}65,73(59,64- \\
71,82 \mathrm{IC} 95 \%)\end{array}$ & - \\
\hline Varón & $60,0 \%$ & $40,0 \%$ & - \\
\hline Mujer & $30,0 \%$ & $70,0 \%$ & $3,5(1,05-11,6$ \\
& & & IC $95 \%)$ \\
\hline NIHSS & $2,21(1,70-2,72$ & $4,92(3,04-6,80$ & - \\
& $\mathrm{IC} 95 \%)$ & IC 95\%) & $91,7 \%$ \\
\hline Sí antecedentes personales de depresión & $8,3 \%$ & & $16,86(1,96-$ \\
& & $70,6 \%$ & $3,25(0,932-$ \\
\hline Con síntomas depresivos previos al ictus & $29,4 \%$ & & $11,380$ IC $95 \%)$ \\
\hline
\end{tabular}

Para explicar la depresión, se construyó un modelo de regresión logística. En este análisis, la variable sexo femenino no resulta significativa pero, en cambio, sí lo son las variables gravedad- NIHSS y tener antecedentes personales de depresión. De hecho, los resultados apuntan a que lo que mayor riesgo confiere para tener depresión postictus es tener antecedentes personales de depresión.

Tabla 2

Cálculo de regresión logística con las variables significativas en el estudio univariado.

\begin{tabular}{|l|c|c|c|}
\hline & OR & $\begin{array}{c}\text { IC 95\% } \\
\text { Inferior }\end{array}$ & $\begin{array}{c}\text { IC 95\% } \\
\text { Superior }\end{array}$ \\
\hline Tener antecedentes personales de depresión & 25,79 & 1,52 & 437,04 \\
\hline NIHSS & 1,63 & 1,04 & 2,55 \\
\hline Ser mujer & 1,25 & 0,24 & 6,38 \\
\hline
\end{tabular}

* Controlado por tener antecedentes personales de depresión, sexo, nivel de NIHSS, síntomas depresivos previos al ictus y edad. 
ORIGINALES Y REVISIONES

En cuanto al perfil clínico de los cuadros depresivos, se observó que los síntomas depresivos presentados por hombres eran similares a los presentados por mujeres, encontrándose en ambos casos que el síntoma predominante era la tristeza $(70 \%)$ frente a la apatía/falta de interés hallada en otros estudios; otros síntomas frecuentes que hallamos son: tensión interior $(60 \%)$, sueño reducido (44\%) y dificultades de concentración (36\%). La constelación de síntomas era también prácticamente la misma independientemente de la gravedad del cuadro depresivo (depresión menor vs. depresión mayor).

Por último, encontramos que más de la mitad de los pacientes atribuían sus síntomas depresivos a distintas causas, entre las que encontramos: a) circunstancias vitales previas al ictus tales como el fallecimiento de un ser querido, ser separado del cónyuge tras el ingreso de éste en una residencia de ancianos, problemas familiares o conyugales, enfermedad de hijos, haber sufrido un accidente de tráfico semanas antes del ictus, etc. b) al propio cuadro depresivo que decían sufrir de antes de ocurrir el ictus y por el cual ya estaban en tratamiento (este caso sólo se dio en tres pacientes de la muestra: dos mujeres con depresión moderada y un hombre con depresión leve); y c) verse hospitalizados, lejos de su familia, preocupados por no poder cuidar de los suyos, por tener asuntos que resolver, depender de otros (para su aseo, para alimentarse, para que se hagan cargo de asuntos pendientes), etc.

\section{Discusión.}

La mayoría de los estudios sobre la depresión en la fase aguda del ictus, se centran en el primer mes siguiente al mismo y son muy escasos los estudios que estudian la depresión postictus en los primeros días después del ictus. Los datos que hemos obtenido en nuestro estudio reflejan el cuadro depresivo en los 4-6 primeros días tras el ictus isquémico.

En nuestro estudio, observamos que sólo 7 pacientes no presentaban ningún tipo de sintomatología depresiva; 17 sujetos presentaban síntomas depresivos pero si constituir un cuadro depresivo completo, 19 pacientes presentaban depresión leve y 7 depresión moderada. En ningún caso se dió depresión grave a pesar de que, en muchos pacientes, el nivel de gravedad del ictus isquémico era elevado; de hecho, aunque la gravedad- NIHSS resultaba significativa en su relación con la probabilidad de presentar depresión postictus, no se halló relación entre el nivel de NIHSS y la gravedad del cuadro depresivo. Asimismo, al igual que en otros estudios, tampoco hallamos asociación entre localización y extensión del ictus isquémico y tener depresión (6;7). Estos resultados nos induce a 
cuestionar la teoría biológica sobre la etiopatogenia de la depresión postictus, inclinándonos más por una hipótesis psicológica. Así, nos planteamos la posibilidad de explicar la depresión postictus desde la perspectiva de una reacción de duelo como trastorno adaptativo en respuesta a una situación vital estresante, e interpretar la clínica depresiva como una reacción psicológica ante una situación de discapacidad que pone a la persona en una situación de dependencia.

Encontramos que los factores asociados a una mayor vulnerabilidad a presentar depresión postictus son el nivel de NIHSS (lo que implica pérdida de salud y de capacidad funcional), ser mujer y tener antecedentes personales de depresión, factores que, como se ha podido comprobar en otros estudios $(20 ; 21)$ también se encuentran presentes en la depresión funcional. Así, entre los factores determinantes de un duelo, se encuentran, además de una pérdida significativa, el sexo (mayor implicación emocional de las mujeres en el proceso de duelo) y tener antecedentes personales de trastornos afectivos (las personas con antecedentes personales de depresión suelen sufrir más durante el proceso de duelo) $(14 ; 22)$. Es más, en nuestro estudio, aunque la variable "síntomas depresivos previos al ictus" no resultó significativa, los datos sugieren una relación entre ser mujer y tener síntomas depresivos previos al ictus con una mayor probabilidad de sufrir depresión postictus.

Por otra parte, en nuestra muestra encontramos que la clínica depresiva predominante era la tristeza junto con tensión interior y alteraciones del sueño, independientemente de la gravedad del cuadro depresivo, del sexo y del nivel de NIHSS. Se sabe que, en el duelo, el sentimiento más comúnmente encontrado es la tristeza y, como reacciones somáticas, son frecuentes el insomnio, la pérdida de apetito y tensión interior. Ninguno de los pacientes deprimidos expresaron sentimientos de autodescalificación personal y sólo dos (un hombre y una mujer, ambos con depresión moderada) refirieron ideación suicida, síntomas muy frecuentes en lo que se considera un trastorno depresivo mayor y muy infrecuentes en las reacciones de duelo no patológico (22).

Finalmente, en un trastorno depresivo mayor la persona afectada suele no encontrar motivo para su pena mientras que, en una reacción de duelo, siente que su aflicción está plenamente justificada. En la muestra de nuestro estudio, encontramos que la mayoría de los pacientes deprimidos atribuían su estado de ánimo a hechos concretos fácilmente identificables: bien a las consecuencias derivadas de haber enfermado, lo cual implica pérdidas concurrentes (permanecer lejos del hogar familiar, pérdida de autonomía, del rol de cuidador de otros...), bien a acontecimientos vitales graves en el pasado reciente que implicaban pérdidas (separación del cónyuge, divorcio, fallecimiento de un familiar cercano...), factores que también se han podido observar en otras investigaciones $(4 ; 11)$. 
ORIGINALES Y REVISIONES

\section{Conclusión.}

En nuestro estudio, encontramos que la clínica depresiva hallada se asemeja mucho a la depresión característica de una reacción adaptativa de duelo. Constituyendo un duelo la reacción emocional y proceso psicológico subsiguiente a una pérdida significativa, podemos entender que implica también la reacción ante la pérdida de salud y/o capacidad funcional. Y siendo así el duelo una respuesta normal del ser humano ante situaciones de pérdida, en tanto no se complique por extensión temporal y/o agravamiento de los síntomas, tal vez debiéramos plantearnos los cuadros depresivos en personas con ictus isquémico desde una óptica que no patologice lo que es una reacción adaptativa lógica ante la enfermedad y sus consecuencias. Esto implicaría asimismo replantearnos las pautas de tratamiento en estos casos.

No obstante, para poder argumentar de forma más sólida la hipótesis de una reacción de duelo, se hace necesario continuar en esta línea de investigación ampliando a distintos periodos temporales del ictus isquémico. 


\section{BIBLIOGRAFÍA:}

(1) Robertson M.M; Katona C.L.E (Eds). "Depresión y enfermedades somáticas". En: Perspectivas en Psiquiatría. Vol. 6. Edit. Wiley, 1998.

(2) BACA BALDOMERo, E. "Aspectos psiquiátricos de las alteraciones vasculares" cerebrales. En: Martínez Lage J.M; Khachaturian ZS (Eds). Alzheimer XXI: Ciencia y sociedad. Madrid, Edit. Masson, 2001.

(3) Olivera, FJ., "Relación entre patología vascular y depresión: desde el ACV hasta la depresión vascular”, Rev Psicogeriatría, 2005, 5, pp. 34-36.

(4) Hackett, ML., y OtRos, "Frequency of depression after stroke. A Systematic Review of Observational Studies”. Stroke, 2005, 36, pp. 1330-1340.

(5) CARson, JA., Y otros, "Depression after stroke and lesion location: a systematic review". Lancet, 2000, 356, pp. 122-127.

(6) Carota, A.; Staub, F.; Bogousslavsky, J., "Emotions, behaviours and mood changes in stroke". Curr Opin Neurol, 2002, 15, pp. 57-69.

(7) CAEIRO, L., Y Otros, "Depression in acute stroke", J Psychiatry Neuroscience, 2006, 31, pp. 377-383.

(8) Burvill, P., Y OtRos, "Risk factors for postroke depression". Int J Geriatr Psychiatry 1997, 12 (2), pp. 219-226.

(9) Lieberman, D., Y OtRos, "Characterization of elderly patients in rehabilitation: stroke vs. hip fracture”, Disabil Rehabil, 1999; 21: 542-547.

(10) LIPSEY, JR., Y OTROS. "Phenomenological comparision of poststroke depression and functional depression", Am J Psychiatry, 1986, 143, pp. 527-529.

(11) Hackett, ML.; Anderson, CS., "Predictors of depression after stroke. A Systematic Review of Observational Sudies”, Stroke, 2005, 36, pp. 2296-2301.

(12) Neymeyer, Robert A. Aprender de la pérdida. Una guía para afrontar el duelo. Barcelona, Ediciones Paidós Ibérica, 2007.

(13) BAYÉs, R. Psicología del sufrimiento y de la muerte. Barcelona, Edit. Martínez Roca, 2001.

(14) Сово Medina, C. El valor de vivir. Elogio y razón del duelo. Madrid, Ediciones Libertarias, 1999 .

(15) Organización Mundial de la Salud. The ICD-10 Classification of Mental and Behavioural Disorders: Diagnostic criteria for research. Edit: Meditor, 1992.

(16) Starkstein, SE.; Robinson, RG., "Neuropsychiatric aspects of cerebral vascular disorders". En: The American Psychiatric Press Textbook of Neuropsychiatry. Washington, American Psychiatric Press, 1992.

(17) House, A., "Depression associated with stroke", J Neuropsychiatry, 1996, 8, pp. 453-457.

(18) Gainotti, G., Y отRos, "The Post-Stroke Depression Rating Scale: a test specifically devised to investigate affective disorders of stroke patients", J Clin Exp Neuropsychol, 1997, 19, pp. 340-356.

(19) Gainotti, G.; Azzoni, A.; Marra, C., "Frequency, phenomenology and anatomical-clinical correlates of major poststroke depression". Br J Psychiatry 1999, 175, pp. 163-167.

(20) KendLER, K., Y OTROS, "The prediction of major depression in women: towards an integrated etiologic model”, Am J Psychiatry, 1993, 150, pp. 1139-1148. 
ORIGINALES Y REVISIONES

(21) Sharpe, M., Y OtRos, "Depressive disorders in long-term survivors of stroke: associations with demographic and social factors, functional status, and brain lesion volume", Br J Psychiatry, 1994, 164, pp. 380-386.

(22) Worden, J.W. El tratamiento del duelo. Asesoramiento psicológico y terapia. Barcelona, Ediciones Paidós Ibérica, 2004.

* M $^{\mathrm{a}}$ Gema Fernández Gómez. Psicóloga Interno Residente de tercer año (PIR-3). Servicio de Psiquiatría del Hospital Universitario de Gran Canaria Dr. Negrín; Ayoze González Hernández. Neurólogo. Servicio de Neurología del Hospital Universitario de Gran Canaria Dr. Negrín. Las Palmas de Gran Canaria.

Correspondencia: Mª Gema Fernández Gómez. Correo electrónico: gofege@ hotmail.com

** Recibido: $1 / 11 / 2008$

*** Los autores quieren agradecer a la Dra. Dolores Fiuza (Unidad de Investigación del Hospital Universitario de Gran Canaria Dr. Negrín) y a la Dra. Petra León (psiquiatra del Servicio de Psiquiatría del Hospital Universitario de Gran Canaria Dr. Negrín) su guía y sugerencias en la elaboración del texto. 